\title{
COMUNICAÇÃO
}

\section{A TEORIA SUBJACENTE À ESCALA WECHSLER DE INTELIGÊNCIA PARA CRIANÇAS (WISC)*}

\author{
THE TEORY BEHIND WECHSLER \\ INTELLIGENCE SCALE FOR CHILDREN
}

Patrícia Waltz SCHELINI**

PUC-Campinas/FAPESP

\begin{abstract}
RESUMO
O objetivo deste trabalho foi discutir teoricamente as concepções de David Wechsler acerca da inteligência, de forma a possibilitar a compreensão de seu mais famoso instrumento de avaliação psicológica infantil: o WISC. Neste sentido, são apresentados e comentados conceitos como a capacidade global e aspectos cognitivos e conativos da inteligência.
\end{abstract}

Palavras-chave: WISC, inteligência, David Wechsler

\begin{abstract}
The purpose of this study was to discuss the David Wechsler's conceptions about intelligence, to make possible the comprehension of his most famous children psychological evaluation test: WISC. Therefore, intelligence concepts as global capacity and cognitive and conative aspects. Were presented and commented.
\end{abstract}

Key words: WISC, intelligence, David Wechsler

A história do WISC foi iniciada na década de trinta quando David Wechsler, trabalhando como psicólogo no hospital Bellevue de Nova lorque, constatou a necessidade de um instru-

\footnotetext{
(*) Agência financiadora: FAPESP.

${ }^{(*)}$ Endereço: Av.: Diogo Álvares, 1876, Parque São Quirino - CEP: 13089-000 - Campinas, São Paulo. Tel.: (19) 32561028 E-mail: pwalsche@hotmail.com
} 
mento que avaliasse a inteligência de seus clientes adultos; já que os testes até então existentes, como o Stanford-Binet, eram direcionados à população infantil (Butcher, 1981; Cronbach, 1996).

Após vários estudos, incluindo análises cuidadosas dos testes já padronizados, Wechsler criou, em 1939, a Escala de Inteligência Wechsler-Bellevue, destinada à avaliação intelectual de adultos (Wechsler, 1944).

Em meados da Segunda Guerra Mundial, Wechsler elaborou a forma II da Escala de Inteligência para Adultos (WAIS), a Escala de Inteligência para Crianças (WISC) e a Escala Wechsler de Inteligência para Pré-Escolares (WPPSI).

A primeira edição do WISC, publicada em 1949, foi elaborada como uma extensão, para níveis etários inferiores, da Wechsler-Bellevue, incorporando os itens mais fáceis da mesma, sobretudo em sua forma II.

David Wechsler, a princípio, foi influenciado pelo modelo teórico de Spearman, mas, gradativamente, e a partir de sua experiência clínica, concluiu que a inteligência geral não poderia ser simplesmente equiparada à habilidade intelectual, devendo ser considerada uma manifestação da personalidade. Nesse sentido, a inteligência foi definida por Wechsler como um "agregado ou uma capacidade global do indivíduo para agir intencionalmente, pensar racionalmente e relacionar-sede maneira eficaz com seu ambiente" (Wechsler, 1944, p.3).

O termo "global" foi utilizado porque, de acordo com Wechsler, a inteligência caracterizaria o comportamento inteligente de maneiratotal e seria uma entidade multifacetada, um complexo de diversificados e numerosos componentes. Além disso, não estaria relacionada apenas a habilidades específicas, envolvendo tanto aspectos cognitivos quanto componentes afetivos e conativos-os chamados aspectos não-intelectivos (Wechsler, 1944, 1975).
Os elementos não-cognitivos seriam traços de personalidade e atitude que influenciariam a expressão do comportamento inteligente. A motivação, a dependência e independência, a impulsividade, a ansiedade e a persistência exemplificariam esses componentes (Wechsler, 1944, 1950, 1975; Zachary, 1990). Nesse sentido a inteligência não seria apenas a habilidade de aprender, abstrair ou tirar proveito de experiências, mas também uma capacidade de adaptação e realização, de modo que as habilidades intelectuais por si mesmas poderiam ser insuficientes à execução de uma tarefa ou à adaptação ao meio (Wechsler, 1950).

Segundo Wechsler (1940. apud Matarazzo, 1976) a afirmação de que o comportamento inteligente implicaria algo mais que a pura capacidade intelectual, poderia ser justificada através de duas evidências: uma clínica e a outra estatística. A evidência clínica baseava-se no fato de indivíduos com Q.I. idêntico diferirem de maneira relevante quanto à capacidade de lidar com o ambiente. Já a estatística dizia respeito à impossibilidade de explicar mais do que $50 \%$ a $70 \%$ da variância correlacional entre os testes, depois de conhecidos os componentes intelectuais. Assim os $50 \%$ a $30 \%$ da variância fatorial restante somente poderiam estar relacionadas aos componentes não intelectivos.

Ainda em relação aos fatores nãointelectivos, Wechsler $(1944,1950)$ relatou vários estudos cujas conclusões fundamentaram suas concepções sobre a existência de tais fatores. Um desses estudos foi realizado em 1913 por Webb que, através da análise fatorial de uma bateria de testes, isolou o fator "w", relacionado à moral e portanto denominado "escrupulosidade".

Alguns anos depois, Lankese Wynn Jones demonstraram a existência de um outro fator não-intelectivo conhecido como "p", em alusão à perseveração, ou sejaà tendência dos sujeitos resistirem a mudanças. No mesmo sentido, 
Brown, em 1931, discutiu a possibilidade de traços de caráter estarem presentes como fatores em testes de inteligência e Cattell, no ano de 1933, demonstrou correlações entre testes de temperamento e níveis de inteligência.

Entretanto, dentre os diversos estudos, Wechsler $(1944,1950)$ destacou o de W.P. Alexander como o que mais havia contribuído à identificação dos fatores não-intelectivos.

Alexander, analisando a variância intercorrelacional de uma série de testes verbais, de performance e de desempenho acadêmico, constatou que além dos fatores $g$ (geral), $v$ (habilidade verbal) e $\mathrm{p}$ (habilidade prática), outros dois fatores contribuíam significativamente à variância total observada: o fator $x \mathrm{e}$ o fator z. Esses dois novos fatores estariam relacionados ao interesse e à persistência do sujeito na realização de determinadas tarefas, podendo ser descritos como fatores de personalidade ou temperamento que exerceriam importante papel no desempenho acadêmico.

No que diz respeito mais especificamente ao fator $\chi$, este determinaria o interesse individual, relacionando-se não às aptidões, mas ao temperamento; enquanto que o fator $Z$ representaria um aspecto do temperamento relacionado à realização. Vale ressaltar que os fatores $\chi$ e Z poderiam variar consideravelmente de um teste para outro, entretanto a presença de ambos foi constatada por Alexander na maioria dos instrumentos pesquisados.

A elucidação do significado e importância dos fatores não-intelectivos é fundamental à compreensão da inteligência como uma capacidade global. Entretanto, além de global, Wechsler também definiu-a como uma espécie de "agregado", porque, de acordo com suas concepções, seria composta por várias habilidades, as quais, apesar de não inteiramente independentes, seriam qualitativamente diferenciadas. O termo "agregado", porém, não deve remeter à falsa idéia de que Wechsler consideravaa inteligência como uma merasoma de habilidades, mesmo porque o comportamento inteligente não seria apenas uma função do número ou dos tipos de habilidades, mas também da maneira pela qual estas habilidades estariam combinadas (Wechsler,1944).

Os pressupostos de Wechsler levaramno à certeza de que tarefas estandartizadas, como o próprio WISC, não seriam capazes de avaliar de maneira total a capacidade intelectual, podendo, contudo, mensurar os principais aspectos da inteligência e fornecer confiáveis índices da capacidade global de cada indivíduo (Wechsler, 1991).

Ao mesmo tempo em que reconhecia a impossibilidade dos instrumentos avaliarem a inteligência em sua totalidade, Wechsler (1944) mencionou que os testes mensuravam não só capacidades de aprendizagem, raciocínio ou mesmo a intelectual geral, mas também outras que não poderiam ser definidas como puramente cognitivas ou intelectivas: as habilidades relacionadas aos fatores não-intelectivos, como o "X" e o "Z", já citados anteriormente.

Nesse sentido quanto mais fossem excluídos os fatores não-intelectivos de um teste menor seria a sua eficácia na avaliação da inteligência. Daí a necessidade da elaboração de instrumentos nos quais tais fatores estivessem claramente presentes (Wechsler, 1944, 1949).

É válido ressaltar que Wechsler, ao enfatizar a importância da presença de fatores não-intelectivos nos testes de inteligência, não pretendeu afirmar que esses instrumentos avaliariam a personalidade, mas sim que continham elementosque eram essencialmente fatores de personalidade. Desse modo, conforme exemplificou o próprio Wechsler (1950), um neurótico poderia ter um desempenho inferior à média no subteste Números não por uma dificuldade de memorização, mas devido à ansiedade mobilizada pelo teste. Ou seja, o desempenho do sujeito receberia interferências dos fatores não-intelectivos, mesmo porque a inteligência 
estaria contida ou faria parte de uma estrutura maior, a personalidade.

Da constatação de que as atividades padronizadas para a avaliação da capacidade intelectual não poderiam mensurar de maneira total a inteligência, talvez decorram considerações como as de Matarazzo (1990) e Mettrau \& Almeida (1995), referentes ao fato de que a avaliação da inteligência não deveria ficar restrita aos dados obtidos pelo uso de testes, cabendo ao psicólogo considerar as informações da história devida do sujeito (antecedentes sociais e culturais, processo de desenvolvimento, informações da escola...) e suas próprias observações.

Abordadas as principais noções de Wechsler acerca da inteligência, torna-se necessário esclarecer que sua teoria de inteligência não poderia deixar de ser considerada como uma teoria fatorialista, mesmo que a ênfase não tenha sido a descrição minuciosa dos fatores representativos das capacidades intelectuais.

Nesse sentido, apesar de ter definido a inteligência como um constructo unitário, global, o modelo teórico de Wechsler seria, de acordo com Zachary (1990), implicitamente hierárquico e, portanto, fatorial. Ainda que Wechsler não tenha tido a intenção de formular uma teoria hierárquica e criar um instrumento que avaliasse a inteligência de acordo com tal modelo, vários tipos de análises têm confirmado um suporte hierárquico subjacente às concepções de Wechsler e, conseqüentemente, às suas escalas.

Uma dessas análises foi feita por Kaufman (1975) que, analisando os dados de padronização da Wechsler Intelligence Scale for Children-Revised (WISC-R), verificou a existência de três fatores: Compreensão Verbal, Organização Perceptual e Resistência à Distrabilidade.

O fator de Compreensão Verbal estaria associado a um constructo que Wechsler pretendia avaliar através da Escala Verbal; o mesmo ocorrendo entre o fator de Organização Perceptual e a Escala de Execução. Já o fator de Resistência à Distrabilidade corresponderia a um atributo comportamental (Kaufman \& Reynolds, 1983), sendo considerado um fator menor ou menos relevante que os dois anteriores (Kaufman, Harrison \& Ittenbach, 1990). Um quarto fator, denominado "Velocidade de Processamento", foi isolado através do método de análise fatorial aplicado nos subtestes do WISC III (Wechsler, 1991).

Além desses quatro fatores, estudos acerca dos subtestes das Escalas Wechsler, como o realizado por Wallbrown, Blaha \& Wherry (1974), comprovaram a existência de um fator global de inteligência. De acordo com Zachary (1990), o modelo implicitamente hierárquico de Wechsler seria representado pelo fator global no ápice e pelos fatores verbal e de performance num nível secundário.

As tentativas de isolamento de fatores contribuíram, de acordo com Cunha (1993), para um melhor entendimento dos escores resultantes da aplicação das Escalas criadas por Wechsler.

Finalmente, é válido ressaltar que o principal objetivo do presente estudo foi possibilitar o entendimento dos aspectos teóricos referentes à Escala Wechsler de Inteligência para Crianças (WISC), de modo que os profissionais tenham consciência de que um instrumento adequado sempre deve ser alicerçado em uma teoria. Ou seja, um constructo apenas pode ser mensurado, testado apropriadamente, se antes for definido e exaustivamente compreendido.

\section{REFERÊNCIAS}

BUTCHER, H.J.(1981). A inteligência humana - natureza e avaliação. São Paulo: Perspectiva.

CRONBACH, L.J.(1996).Fundamentos da testagem psicológica. 5a ed. Porto Alegre: Artes Médicas. 
CUNHA, J.A.(1993). Psicodiagnótico-R. 4ª ed. Porto Alegre: Artes Médicas.

KAUFMAN, A.S. (1975) Factor analysis of the WISC-R at eleven age (evels between 6.5 and 16.5 years. Journal of Consulting and Clinical Psychology, 43: 135-147.

KAUFMAN,A.S \& Reynolds, C.R. (1983). Clinical evaluation of intellectual function. In Weiner, I.b. (ed.) Clinical methods in psychology. New York: Wiley \& Sons. pp. 100-151.

KAUFMAN,A.S., Harrison, P.L. \& Ittenbach, R.F. (1990). Intelligence testing in the schools. In Gutkin, T.B. \& Reynolds, C.R. (ed). The Handbook of School Psychology. $2^{\text {nd }}$. New york: John Wiley \& Sons. Pp. 289-327.

MATARAZZO, J.D. (1976). Wechsler: Medida e avaliação da inteligência do adulto. São Paulo: Manole.

MATARAZZO, J.D. (1990). Psychological assessment versus psychological testing: Validation trem binet to the school, clinic and coutrooms. American Psychologist, 45: 999-1017.

METTRAU, M.B. \& Almeida, L.S.(1995). Inteligência: Visualizar formas mais globais para a sua avaliação. In Almeida, L.S. \&
Ribeiro, I.S. (org.). Avaliação psicológica: Formas e contextos. Vo1.3. Braga: APPORT. pp.435-444.

WALLBROWN,F.H, Blaha, J. \& Wherry, J. (1974). The hierarchichal factor structure of the Wechsler Adult Intelligence Scale. British Journal of Educational Psychology, 44: 47-56.

WECHSLER, O. (1944). The measurement of adult intelligence. $3^{\text {th }}$ ed. Baltimore: The Williams \& Wilkins Company.

WECHSLER, D.(1949). Wechsler Intelligence Scale for Children: Manual. New York: The Psychological Corporation.

WECHSLER, 0.(1950). Cognitive, conative and non-intellective intefligence. American Psychologist, 15: 78-83.

WECHSLER, 0.(1975). Intelligence defined and undefined: A relativistic appraisal. American Psychologist, 30(2): 135-139.

WECHSLER, O. (1991). Wechsler Intelligence Scale for Children-Revised: Manual. San Antonio: Psychological Corporation.

ZACHARY, R.A. (1990). Wechsler's Intelligence Scales: Theoretical and practical considerations. Journal of Psychoeducational Assessment, 8: 276-289. 